\title{
Temperature-independent Optical Fiber Liquid Level Sensor Based on an In-fiber Mach-Zehnder Interferometer Formed by Cascaded Polarization-maintaining Long-period Fiber Gratings
}

\author{
Sungwook Choi, ${ }^{1}$ Do Kyung Kim, ${ }^{1}$ Jihoon Kim, ${ }^{2}$ \\ Seul-Lee Lee, ${ }^{1}$ Min Seok Kim, ${ }^{1}$ and Yong Wook Lee ${ }^{1,2^{*}}$ \\ ${ }^{1}$ Interdisciplinary Program of Biomedical Mechanical and Electrical Engineering, Pukyong National University, \\ 45 Yongso-ro, Nam-gu, Busan 48513, Korea \\ ${ }^{2}$ School of Electrical Engineering, Pukyong National University, \\ 45 Yongso-ro, Nam-gu, Busan 48513, Korea
}

(Received June 22, 2019; accepted September 24, 2019)

Keywords: optical fiber sensor, liquid level sensor, long-period fiber grating, polarization-maintaining fiber

Here, we propose an optical fiber liquid level sensor (OFLLS) based on a Mach-Zehnder interferometer (MZI) formed with cascaded long-period fiber gratings (LPFGs) written on a polarization-maintaining fiber (PMF). The proposed sensor consists of a broadband light source, a polarization controller, 83.4-mm-long cascaded LPFGs as a sensor head, and an optical spectrum analyzer. Each LPFG was fabricated using a $\mathrm{CO}_{2}$ laser with a grating period of $490 \mu \mathrm{m}$ and a grating length of $14.2 \mathrm{~mm}$. The grating-free fiber segment (GFFS) between two LPFGs, which serves as an active area of the sensor head, was $55.0 \mathrm{~mm}$ long. If the external refractive index of the active area is varied by increasing the amount of water contained in a measuring cylinder within which the sensor head is located along its longitudinal direction, the refractive index difference between air and water causes an additional phase shift in the recoupled cladding mode. This additional phase shift is responsible for a wavelength shift in the in-fiber interference created by the cascaded LPFGs. Owing to PMF birefringence, the phase matching condition for the LPFG and thus the in-fiber interference are all polarizationdependent. Our cascaded LPFGs fabricated on the PMF show different interference spectra for two orthogonal input polarization states, e.g., linear horizontal polarization (LHP) and linear vertical polarization (LVP). For LHP and LVP input signals, the water level sensitivity was measured as approximately -37.7 and $-137.0 \mathrm{pm} / \mathrm{mm}$ in a measurement range from 0 to $55 \mathrm{~mm}$, respectively. Moreover, the cascaded LPFGs are also sensitive to ambient temperature changes, and temperature sensitivities at LHP and LVP input signals were measured as 26.8 and $\sim 209.0 \mathrm{pm} /{ }^{\circ} \mathrm{C}$ in a temperature range from 30 to $70{ }^{\circ} \mathrm{C}$, respectively. Linear and independent responses to water level and ambient temperature changes enable our sensor to simultaneously measure water level and ambient temperature variations.

*Corresponding author: e-mail: yongwook@pknu.ac.kr https://doi.org/10.18494/SAM.2020.2477 


\section{Introduction}

Since the fiber grating technology was first reported in $1978,{ }^{(1)}$ fiber gratings have received much attention as optical devices for optical fiber sensors and communications owing to their unique characteristics such as high sensitivity, resistance to corrosion, and immunity to electromagnetic interference. With these characteristics, fiber grating sensors have been reported to be capable of measuring strain, temperature, pressure, refractive index, liquid level, and so forth. ${ }^{(2-6)}$ Among them, fiber grating liquid level sensors have been in high demand in a variety of industrial applications such as fuel storage and water level monitoring. ${ }^{(7,8)}$ Generally, conventional fiber Bragg gratings (FBGs) have been widely used to measure strain or temperature. However, conventional FBG sensors cannot detect changes in surrounding refractive index (SRI), because light introduced into FBGs is only guided within the core covered by the cladding. Thus, conventional FBGs cannot be utilized for water level measurement. This is why many researchers have used etched or side-polished FBGs for water level measurement, which can detect SRI changes by exposing the evanescent field to water outside the FBGs. ${ }^{(9,10)}$

Yun et al. proposed an optical fiber liquid level sensor (OFLLS) by employing an etched FBG with a grating length of $24 \mathrm{~mm} .{ }^{(9)}$ Since the clad region of the FBG was etched by hydrofluoric acid, the transmittance of the Bragg resonance dip changed according to the difference in refractive index between air and liquid around the grating element, and the water level sensitivity of the dip transmittance was obtained as $\sim 2.54 \mathrm{~dB} / \mathrm{mm} .{ }^{(9)}$ Chang et al. reported an OFLLS based on a 7-mm-long chirped FBG (CFBG). ${ }^{(11)}$ Likewise in the case of the OFLLS based on the etched FBG, the CFBG was etched by hydrofluoric acid to measure $12 \mu \mathrm{m}$ in thickness. They measured the change in liquid level by observing a wavelength shift in the Bragg reflection spectrum, which was induced as the shorter grating period region of the CFBG was gradually immersed in the liquid, and the water level sensitivity of the CFBG was obtained as $\sim 1214 \mathrm{pm} / \mathrm{mm}$. However, despite achieving a very high sensitivity, the etched sensor heads are not suitable for practical industrial applications owing to their very low mechanical strength. Moreover, to broaden its measurement range, the length of the fiber grating should be increased in proportion to the measurement range. However, long fiber gratings are not cost-effective and vulnerable to transverse stress.

A long-period fiber grating (LPFG) is a fiber structure whose core refractive index is modulated in a period ranging from tens to hundreds of micrometers so that light traveling only in the core mode, which satisfies a phase matching condition, is coupled into several cladding modes when it passes through the grating. ${ }^{(12)}$ Owing to this coupling nature between core and cladding modes, LPFGs have been widely used to detect SRI changes with high sensitivity ${ }^{(13)}$ and also been utilized to detect changes in liquid level. ${ }^{(14)}$ In particular, LPFGs are even cost-effective because their fabrication process is much simpler than that of FBGs. In 2011, an OFLLS based on an in-fiber Mach-Zehnder interferometer (MZI) was proposed by Fu et al. using cascaded $3 \mathrm{~dB}$ LPFGs. ${ }^{(15)}$ In the cascaded $3 \mathrm{~dB}$ LPFGs, light is guided in the cladding region of a single-mode fiber (SMF) on which LPFGs are written at the grating-free fiber segment (GFFS) between two 3 dB LPFGs. This cladding mode can react sensitively 
to changes in the SRI of the GFFS and cause an additional phase shift in the cladding mode recoupled into the core mode at the second LPFG. Therefore, when the GFFS is immersed in liquid, the wavelength of the interference spectrum created by the in-fiber MZI shifts owing to the refractive index difference between air $\left(n_{a}=1\right)$ and water $\left(n_{w}=1.333\right)$. The measured sensitivity of the in-fiber MZI-based OFLLS was reported as approximately $-141 \mathrm{pm} / \mathrm{mm}$. However, the temperature cross-sensitivity problem of the cascaded $3 \mathrm{~dB}$ LPFGs was not fully resolved.

Here, we propose a temperature-independent OFLLS based on an in-fiber MZI formed by cascaded $3 \mathrm{~dB}$ LPFGs inscribed on a bow-tie-type polarization-maintaining fiber (PMF), which are referred to as cascaded PM-LPFGs hereafter, with a $\mathrm{CO}_{2}$ laser. The proposed OFLLS consists of a broadband light source, a polarization controller, 83.4-mm-long cascaded PMLPFGs as a sensor head, and an optical spectrum analyzer. Each PM-LPFG was fabricated by using $\mathrm{CO}_{2}$ laser pulses with a grating period of $490 \mu \mathrm{m}$ and a grating length of $14.2 \mathrm{~mm}$. The GFFS between two PM-LPFGs, which serves as an active (or sensitive) area of the sensor head for water level measurement, was $55.0 \mathrm{~mm}$ long. Owing to the modal birefringence of PMF, each PM-LPFG has two different phase matching conditions according to two orthogonal input polarization states, e.g., linear horizontal polarization (LHP) and linear vertical polarization (LVP). Thus, our in-fiber MZI, i.e., the cascaded PM-LPFGs, has two totally different interference spectra depending on the LHP and LVP input signals. This implies that the proposed OFLLS has two spectral indicators for water level measurement, which can be obtained for orthogonal input polarization (i.e., LHP and LVP). For LHP and LVP input signals, the water level sensitivity of the proposed sensor was measured as approximately -37.7 and $-137.0 \mathrm{pm} / \mathrm{mm}$ with adjusted $R^{2}$ values of $\sim 0.999$ and $\sim 0.995$ in a water level measurement range from 0 to $55 \mathrm{~mm}$, respectively. In addition, the cascaded LPFGs are also sensitive to ambient temperature changes, and the temperature sensitivities at LHP and LVP input signals were measured as $\sim 26.8$ and $\sim 209.0 \mathrm{pm} / \mathrm{mm}$ with adjusted $R^{2}$ values of $\sim 0.993$ and $\sim 0.996$ in a temperature measurement range from 30 to $70{ }^{\circ} \mathrm{C}$, respectively. Linear and independent responses to water level and ambient temperature changes enable our sensor to simultaneously measure water level and ambient temperature variations. In Sect. 2, the operation principle of our sensor and the fabrication setup of the sensor head will be explained, and the spectral characteristics of the fabricated in-fiber MZI will be described. Then, experimental results and discussion on the water level and temperature responses of our sensor will be presented in Sect. 3. Finally, a brief summary and conclusion will be provided.

\section{Operation Principle and Sensor Head Fabrication}

Owing to its grating period of hundreds of micrometers, an LPFG induces codirectional coupling between the core mode and some cladding modes, resulting in several rejection bands in its transmission spectrum. The resonance wavelength $\lambda_{0}$ of its rejection band can be expressed as

$$
\lambda_{0}=\left(n_{\text {eff }, \text { core }}-n_{\text {eff }, \text { clad }, m}\right) \Lambda,
$$


where $n_{\text {eff,core, }} n_{\text {eff,clad, } m}$, and $\Lambda$ are the effective refractive index of the core mode, the effective refractive index of the $m$ th order cladding mode, and the grating period, respectively. Since the effective refractive indices of the core and cladding modes are dependent on input polarization, $\lambda_{0}$ varies depending on the input polarization as follows.

$$
\begin{aligned}
& \lambda_{0}(\mathrm{LHP})=\left[n_{\text {eff }, \text { core }}(\mathrm{LHP})-n_{\text {eff }, \text { clad }, m}(\mathrm{LHP})\right] \Lambda \\
& \lambda_{0}(\mathrm{LVP})=\left[n_{\text {eff }, \text { core }}(\mathrm{LVP})-n_{\text {eff, }, \text { clad }, m}(\mathrm{LVP})\right] \Lambda
\end{aligned}
$$

In terms of all variables in equations hereafter, LHP and LVP in parentheses imply that parameters are obtained at input polarization states of LHP and LVP, respectively.

When two LPFGs with a resonance dip of $3 \mathrm{~dB}$ are concatenated in series, that is, cascaded $3 \mathrm{~dB}$ LPFGs are formed, $50 \%$ of light at $\lambda_{0}$ traveling in the core is coupled into the cladding mode at the first LPFG. When this cladding mode propagating through the GFFS between the two LPFGs meets the second LPFG, it is recoupled into the core mode. The uncoupled core and recoupled cladding modes, which have different phase delays, generate interference owing to their phase difference at the second LPFG. This phase difference is primarily created at the GFFS and related with the length of the GFFS (i.e., the separation between the two LPFGs), the effective index difference $\Delta n_{\text {eff }}$ between the core and cladding of the GFFS, and the SRI of the GFFS. If the SRI of the GFFS is changed by immersing the cascaded LPFGs in water, the wavelength of the interference spectrum shifts depending on how much part of the GFFS is immersed in water. When the GFFS is immersed in water, the relative phase difference $\varphi$ between the immersed and nonimmersed regions can be expressed by the following equations: ${ }^{(16)}$

$$
\begin{aligned}
& \varphi(\mathrm{LHP})=\phi_{i n}(\mathrm{LHP})-\frac{2 \pi\left\{\Delta n_{\text {eff }}(\mathrm{LHP}) \cdot L+\left[\Delta n_{\text {eff }}^{\prime}(\mathrm{LHP})-\Delta n_{\text {eff }}(\mathrm{LHP})\right] L^{\prime}\right\}}{\lambda_{0}(\mathrm{LHP})}, \\
& \varphi(\mathrm{LVP})=\phi_{i n}(\mathrm{LVP})-\frac{2 \pi\left\{\Delta n_{e f f}(\mathrm{LVP}) \cdot L+\left[\Delta n_{\text {eff }}^{\prime}(\mathrm{LVP})-\Delta n_{\text {eff }}(\mathrm{LVP})\right] L^{\prime}\right\}}{\lambda_{0}(\mathrm{LVP})} .
\end{aligned}
$$

Here, $\phi_{i n}$ is the sum of the phase shifts in both LPFGs, and $\Delta n_{\text {eff }}$ and $\Delta n_{\text {eff }}^{\prime}$ are the effective refractive index differences between the uncoupled core and recoupled cladding modes when the GFFS is exposed to air and water, respectively. $d, L$, and $L^{\prime}$ are the LPFG length, the GFFS length (i.e., the distance between two LPFGs), and the GFFS length immersed in water, respectively. Hence, the wavelength of the interference spectrum of the cascaded LPFGs shifts in proportion to a change in $L^{\prime}$, as can be checked from the following Eqs. (6) and (7).

$$
\lambda_{0}(\mathrm{LHP}) \propto 2 \pi\left\{\Delta n_{\text {eff }}(\mathrm{LHP}) \cdot L+\left[\Delta n^{\prime}{ }_{\text {eff }}(\mathrm{LHP})-\Delta n_{\text {eff }}(\mathrm{LHP})\right] L^{\prime}\right\}
$$




$$
\begin{aligned}
& \lambda_{0}(\mathrm{LVP}) \propto 2 \pi\left\{\Delta n_{\text {eff }}(\mathrm{LVP}) \cdot L+\left[\Delta n_{\text {eff }}^{\prime}(\mathrm{LVP})-\Delta n_{\text {eff }}(\mathrm{LVP})\right] L^{\prime}\right\} \\
& \Delta \lambda_{0}(\mathrm{LHP}) \propto 2 \pi\left[\Delta n^{\prime}{ }_{\text {eff }}(\mathrm{LHP})-\Delta n_{\text {eff }}(\mathrm{LHP})\right] \Delta L^{\prime} \\
& \Delta \lambda_{0}(\mathrm{LVP}) \propto 2 \pi\left[\Delta n^{\prime}{ }_{\text {eff }}(\mathrm{LVP})-\Delta n_{\text {eff }}(\mathrm{LVP})\right] \Delta L^{\prime}
\end{aligned}
$$

As can be seen from Eqs. (4) and (5), for orthogonal input polarization states of LHP and LVP, the interference spectrum of the in-fiber MZI is significantly different because $\Delta n_{\text {eff }}$ and $\Delta n_{\text {eff }}^{\prime}$ are dependent on input polarization. Furthermore, it can also be found from Eqs. (8) and (9) that the wavelength shift of the interference spectrum would be different according to the input polarization. For convenience, it is assumed that LHP and LVP input signals excite the fast and slow axes of the PMF on which the LPFGs are inscribed, respectively, and interference spectra with the maximum contrast are obtained at these two orthogonal input polarization states.

Figure 1 shows a schematic diagram of the proposed OFLLS, which is composed of a broadband source (BBS, Fiberlabs FL7001), a polarization controller (PC, Agilent 8169A), a sensor head, i.e., cascaded PM-LPFGs, and an optical spectrum analyzer (OSA, Yokogawa AQ6370C). The PC used here consists of a rotatable linear polarizer, a rotatable quarter-wave plate (QWP), and a rotatable half-wave plate (HWP). The sensor head was immobilized with epoxy resin on an acrylic plate in the direction perpendicular to the water surface and placed within a water-filled tempered glass cylinder. The tempered glass cylinder was located on a

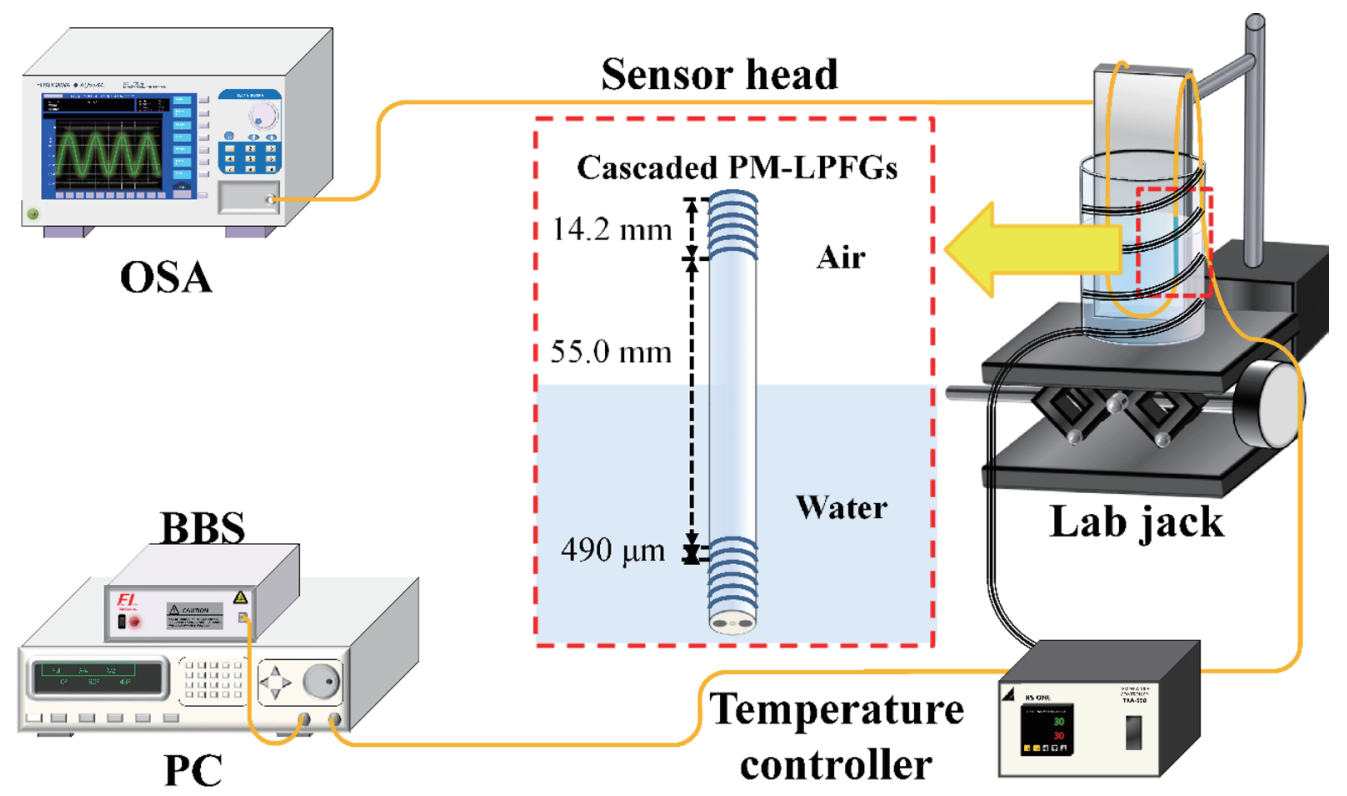

Fig. 1. (Color online) Schematic diagram of proposed OFLLS using in-fiber MZI based on cascaded PM-LPFGs. A red-dashed box shows an enlarged view of the sensor head (i.e., the cascaded PM-LPFGs), which is installed on an acrylic plate in the direction perpendicular to the water surface. 
lab jack so that the sensor head could experience the rise and fall of the water level by adjusting its vertical height. In addition, a heating tape was wound around the glass cylinder to set the desired ambient temperature of the sensor head with a temperature controller. The output light of the BBS is polarized after passing through the PC, and this polarized broadband light is introduced into the sensor head. An interference spectrum is obtained from the in-fiber MZI (i.e., the cascaded PM-LPFGs) and is dependent on input polarization. If the GFFS of the in-fiber MZI is perturbed by SRI or temperature changes, this interference spectrum will be varied (specifically, spectrally shifted), as these perturbations change the phase difference between the uncoupled core mode and the recoupled cladding mode. In particular, this perturbation-induced variation in the interference spectrum also depends on the input polarization of the in-fiber MZI owing to the polarization dependence of the phase difference mentioned above, as predicted in Eqs. (6)-(9).

Figure 2(a) shows a schematic diagram of the fabrication setup of cascaded PM-LPFGs with a $\mathrm{CO}_{2}$ laser (Synrad). By incorporating a 2D laser scanning system based on a $f-\theta$ lens, a bow-tie-type PMF (Fibercore, HB1250T), which was fixed on the fiber holder mounted on a high-resolution motorized linear stage with tension applied to it, was irradiated by $\mathrm{CO}_{2}$ laser pulses. In the cladding region of the PMF, there are stress-applying glass elements that absorb $\mathrm{CO}_{2}$ laser irradiation better than the core of the PMF. Thus, to allow the core of the PMF to sufficiently absorb $\mathrm{CO}_{2}$ laser irradiation, the cross section of the PMF was observed first with a microscope, and then the angular orientation of the PMF was adjusted so that the $\mathrm{CO}_{2}$ laser could illuminate the core of the PMF in the direction of the fast axis of the PMF. The top-left inset of Fig. 2(a) shows the cross section of the PMF used here. The peak power of $\mathrm{CO}_{2}$ laser pulses was $\sim 2.12 \mathrm{~W}$, and the laser beam was scanned 30 times at a grating period of $490 \mu \mathrm{m}$, resulting in a grating length of $\sim 14.2 \mathrm{~mm}$. While the $\mathrm{CO}_{2}$ laser beam was scanned, the transmission spectrum of the fabricated PM-LPFG was monitored by using the BBS connected with the PC and OSA. The three polarization-control elements within the PC, that is, the linear polarizer, QWP, and HWP, were maneuvered so that the transmission spectrum of the fabricated PM-LPFG showed the maximum contrast at its resonance wavelength $\lambda_{0}$. After obtaining a $3 \mathrm{~dB}$ resonance dip in the first PM-LPFG, we moved the PMF by $55.0 \mathrm{~mm}$ in the longitudinal

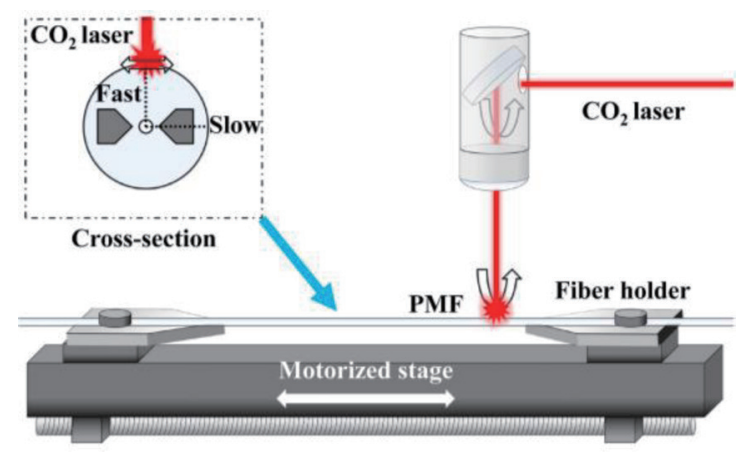

(a)

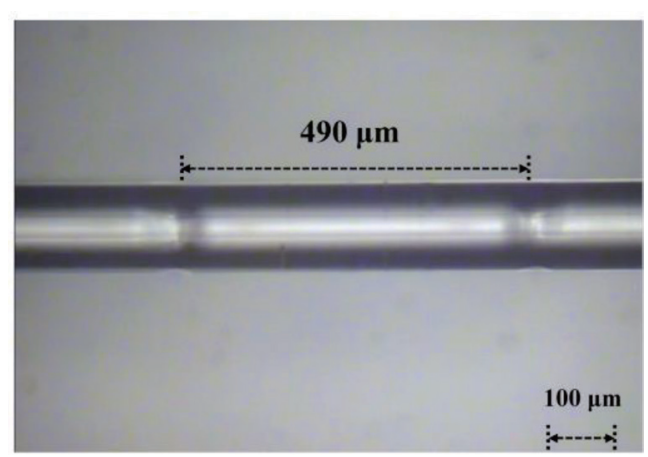

(b)

Fig. 2. (Color online) (a) Schematic diagram of fabrication setup of cascaded LPFGs written on PMF with $\mathrm{CO}_{2}$ laser and (b) side-view microscopy image of part of one LPFG in fabricated cascaded PM-LPFGs. 
direction and inscribed the second LPFG with a $3 \mathrm{~dB}$ resonance dip again. Since the cascaded PM-LPFGs are fabricated with low-power laser pulses and thus little mechanical damage is caused to the PMF, they have mechanical strength similar to that of the conventional PMF. This can facilitate their use in practical applications better than the etched fiber segments. Figure 2(b) shows a side-view microscopy image of the part (specifically, one grating period) of one PM-LPFG in the fabricated cascaded PM-LPFGs. As found from the figure, there is little damage caused by $\mathrm{CO}_{2}$ laser pulses on the PMF segment.

Figure 3 shows the transmission spectra of the fabricated in-fiber MZI, which were measured for various input polarization states without immersing the sensor head in liquid. Polarization angles of 0,45 , and $90^{\circ}$, indicated as black, red, and blue solid lines, imply three input polarization states that excite the fast axis, an axis $45^{\circ}$ to the fast axis, and the slow axis of the PMF comprising the in-fiber MZI, respectively. Here, LHP and LVP correspond to 0 and $90^{\circ}$, respectively. It can be found from the figure that totally different interference spectra are obtained for orthogonal input polarization as predicted above. The visibility of the interference spectrum could be maximized by controlling the QWP and HWP contained in the PC to cancel out the polarization evolution that occurred in the SMF between the PC and the in-fiber MZI. The interference spectra obtained at input polarization angles of $0^{\circ}$ (black) and $90^{\circ}$ (blue) are designated hereafter as fast-axis and slow-axis mode spectra, respectively. Since the wavelength range of the BBS was limited $(<1620 \mathrm{~nm})$, a full interference pattern could not be obtained in the slow-axis mode spectrum compared with the fast-axis one. However, it could be confirmed through water level measurements that there was a similar interference pattern at the wavelength band of $1620 \mathrm{~nm}$ or more. If the fabricated in-fiber MZI is immersed in water, an SRI change (from $n_{a}$ to $n_{w}$ ) causes a change in the effective refractive index difference $\Delta n_{\text {eff }}$, which results in a wavelength shift of the interference spectrum, as can be predicted in Eqs. (6)-(9).

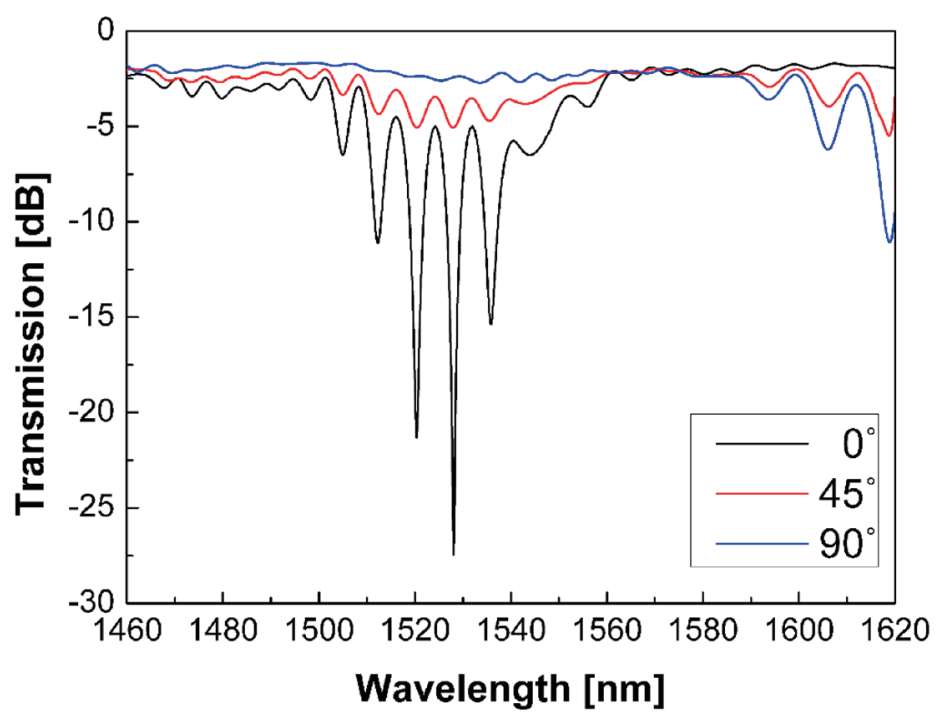

Fig. 3. (Color online) Transmission spectra of fabricated in-fiber MZI, measured for various input polarization states $\left(0,45\right.$, and $90^{\circ}$ linear polarizations) without immersing sensor head in liquid. 


\section{Experimental Results and Discussion}

Figures 4(a) and 4(b) show the variations of the transmission spectrum of the fabricated sensor head at LHP and LVP input polarization states, respectively, when the water level increases from 0 to $55 \mathrm{~mm}$ with a step of $5 \mathrm{~mm}$ at room temperature. As can be seen from the figure, both fast-axis and slow-axis mode interference spectra show blue shifts, i.e., move towards a shorter wavelength region, as the water level increases. A spectral dip, which had the minimum transmittance when the sensor head was not perturbed by SRI changes at room temperature, was determined as an indicator dip for the measurement of the water level response. The wavelength and band rejection ratio (BRR) of the indicator dip were $\sim 1527.99 \mathrm{~nm}$ and $\sim 25.57 \mathrm{~dB}$ in the fast-axis mode spectrum and $\sim 1617.29 \mathrm{~nm}$ and $\sim 7.79 \mathrm{~dB}$ in the slow-axis one, respectively. With these indicator dips at LHP and LVP input polarization states, their wavelength displacements were measured as $\sim 2.06$ and $\sim 7.94 \mathrm{~nm}$ with respect to an increase in water level by $55 \mathrm{~mm}$, respectively. The wavelength displacement of the indicator dip of the slow-axis mode spectrum is $\sim 3.85$ times larger than that of the indicator dip of the fast-axis mode spectrum. This larger wavelength displacement at LVP input polarization is presumed to be caused by a larger $\Delta n_{\text {eff }}$ or $\left(\Delta n_{\text {eff }}^{\prime}-\Delta n_{\text {eff }}\right)$ than the index difference at LHP input polarization. ${ }^{(17)}$

Figure 5 shows the wavelength shifts of indicator dips in the fast-axis and slow-axis mode interference spectra, displayed as blue and red squares, respectively, when the water level increases from 0 to $55 \mathrm{~mm}$ (step: $5 \mathrm{~mm}$ ). Solid lines are linear fits obtained with linear regression. For the fast-axis and slow-axis mode spectra, water level sensitivities of the indicator dips were evaluated as approximately $S_{W L, L H P}=-37.7$ and $S_{W L, L V P}=-137.0 \mathrm{pm} / \mathrm{mm}$, and the adjusted $R^{2}$ values were calculated as $\sim 0.999$ and $\sim 0.995$, respectively. These highly linear water level responses can facilitate the fast and accurate measurement of the water level.

Next, to check the temperature cross sensitivity of the fabricated sensor head, we investigated its temperature response in a temperature range from 30 to $70{ }^{\circ} \mathrm{C}$. Figures 6 (a)

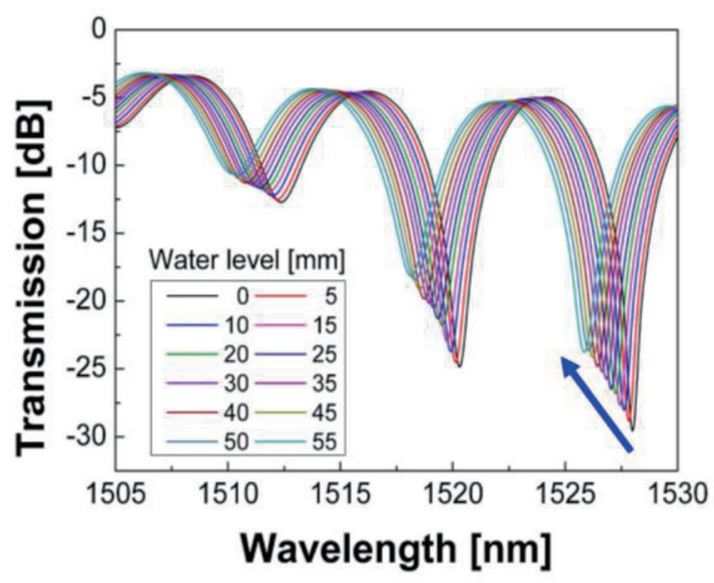

(a)

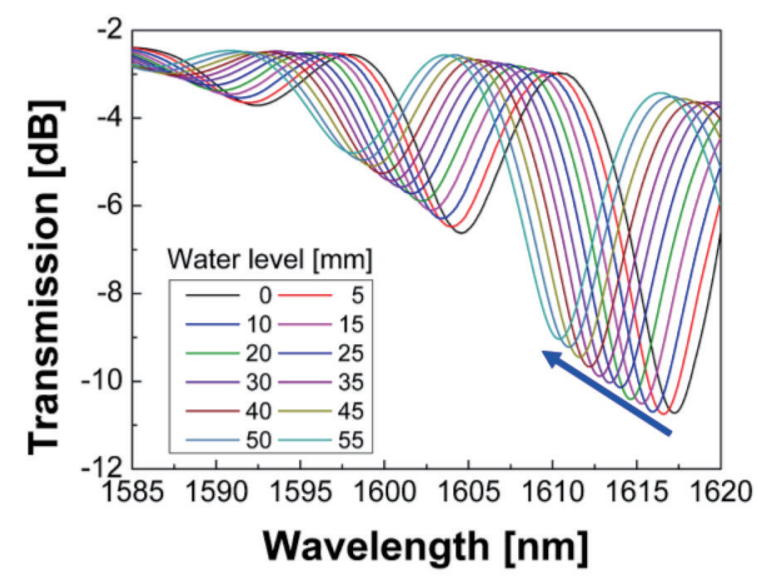

(b)

Fig. 4. (Color online) Variations of transmission spectrum of fabricated sensor head at (a) LHP and (b) LVP input polarization states when water level increases from 0 to $55 \mathrm{~mm}$ with step of $5 \mathrm{~mm}$. 


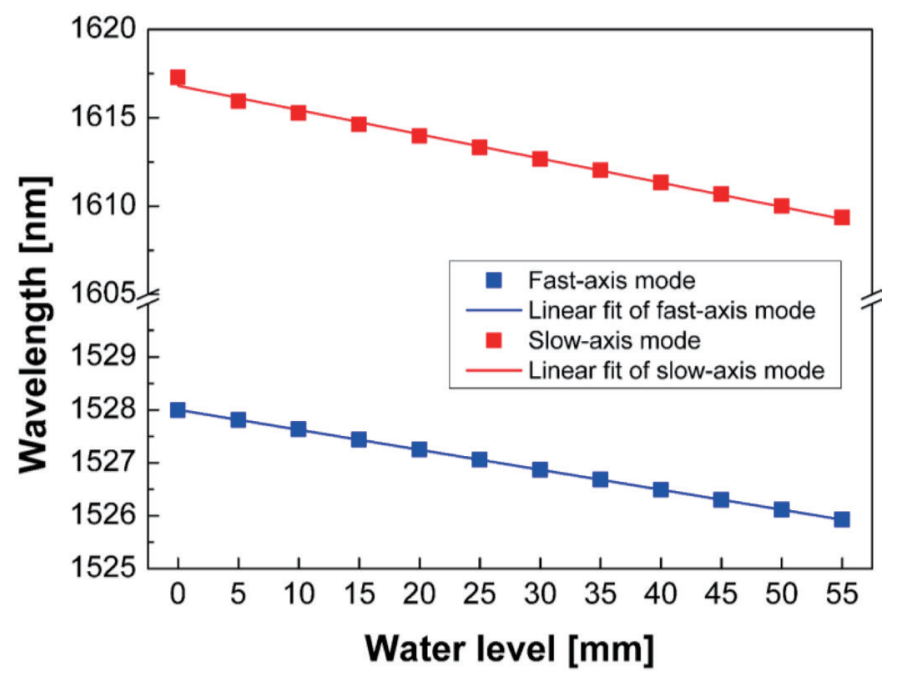

Fig. 5. (Color online) Wavelength shifts of indicator dips in fast-axis and slow-axis mode interference spectra, displayed as blue and red squares, respectively, when water level increases from 0 to $55 \mathrm{~mm}$ (step: $5 \mathrm{~mm}$ ). Solid lines are linear fits obtained with linear regression.

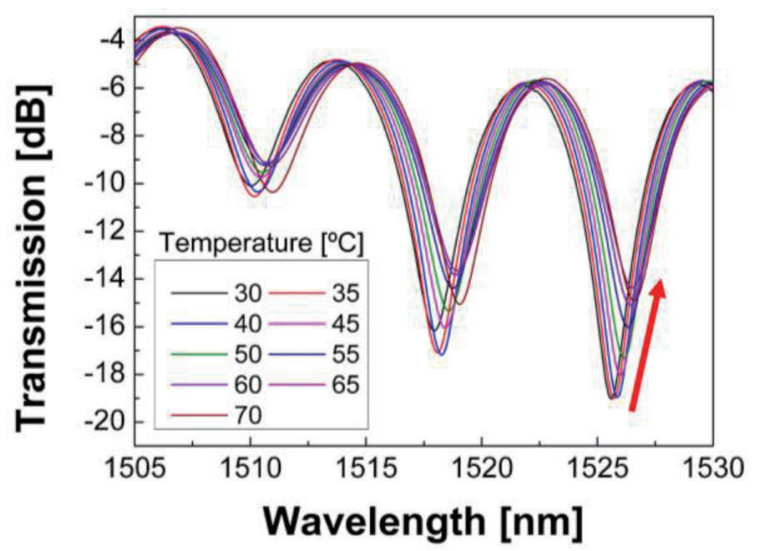

(a)

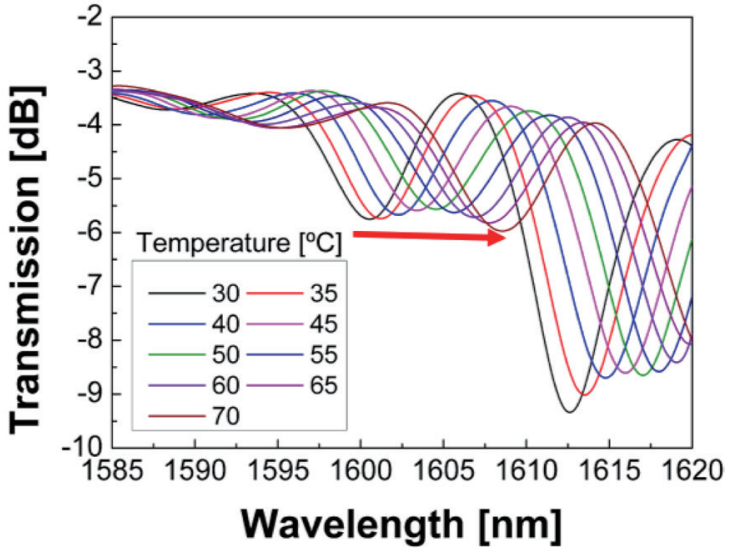

(b)

Fig. 6. (Color online) Variations of the transmission spectrum of fabricated sensor head at (a) LHP and (b) LVP input polarization states when ambient temperature increases from 30 to $70{ }^{\circ} \mathrm{C}$ (step: $5{ }^{\circ} \mathrm{C}$ ) with sensor head completely immersed in water.

and 6(b) show the variations of the transmission spectrum of the fabricated sensor head at LHP and LVP input polarization states, respectively, when the ambient temperature increases from 30 to $70{ }^{\circ} \mathrm{C}$ (step: $5{ }^{\circ} \mathrm{C}$ ) with the sensor head completely immersed in water. In the fast-axis mode interference spectrum (at LHP input polarization), a spectral dip, which had the minimum transmittance at an ambient temperature of $30{ }^{\circ} \mathrm{C}$ when the sensor head was completely immersed in water, was selected as an indicator dip for the measurement of the temperature response. Its wavelength and BRR were $\sim 1525.59 \mathrm{~nm}$ and $\sim 13.17 \mathrm{~dB}$, respectively. Unlike its water level response, the fast-axis mode interference spectrum shows a red shift with increasing 
ambient temperature, and the wavelength displacement of the indicator dip amounts to $\sim 1.04 \mathrm{~nm}$ for an increase in ambient temperature by $40{ }^{\circ} \mathrm{C}$. In the case of the slow-axis mode interference spectrum (at LVP input polarization), a spectral dip with the second minimum transmittance was selected as an indicator dip, because the minimum transmittance dip went beyond the measurement window at an ambient temperature above $60{ }^{\circ} \mathrm{C}$. The selected indicator dip has a BRR of $\sim 2.35 \mathrm{~dB}$ at $\sim 1600.67 \mathrm{~nm}$. Likewise in the case of the fast-axis mode spectrum, the indicator dip also exhibits a red shift with the increase in ambient temperature. Its wavelength displacement amounts to $\sim 7.95 \mathrm{~nm}$ for a temperature increase of $40{ }^{\circ} \mathrm{C}$, which is $\sim 7.64$ times larger than the wavelength displacement of the indicator dip in the fast-axis mode spectrum. For both water level (SRI) and temperature responses, a marked difference in wavelength displacement between orthogonal input polarization states can be explained as follows. Our cascaded PM-LPFGs form a polarization-dependent in-fiber MZI, which has two different interference spectra (i.e., fast-axis and slow-axis mode interference spectra) for orthogonal input polarization states aligned along the slow and fast axes of the PMF. In this in-fiber MZI, each fringe spectrum with multiple dips is created by the interference between the uncoupled core mode and the cladding mode recoupled into the core mode at the second PM-LPFG. The order of this cladding mode can be different depending on input polarization due to the PMF birefringence, ${ }^{(17)}$ and two indicator dips in fast-axis and slow-axis mode interference spectra with different cladding-mode orders can have different responses to SRI or temperature. ${ }^{(18)}$

Figure 7 shows the wavelength shifts of the indicator dips in the fast-axis and slowaxis mode interference spectra, displayed as blue and red circles, respectively, when the ambient temperature increases from 30 to $70{ }^{\circ} \mathrm{C}$ (step: $5{ }^{\circ} \mathrm{C}$ ). Solid lines are linear fits obtained with linear regression. For the fast-axis and slow-axis mode spectra, temperature sensitivities were evaluated as $S_{T, L H P}=\sim 26.8$ and $S_{T, L V P}=\sim 209.0 \mathrm{pm} /{ }^{\circ} \mathrm{C}$, and the adjusted

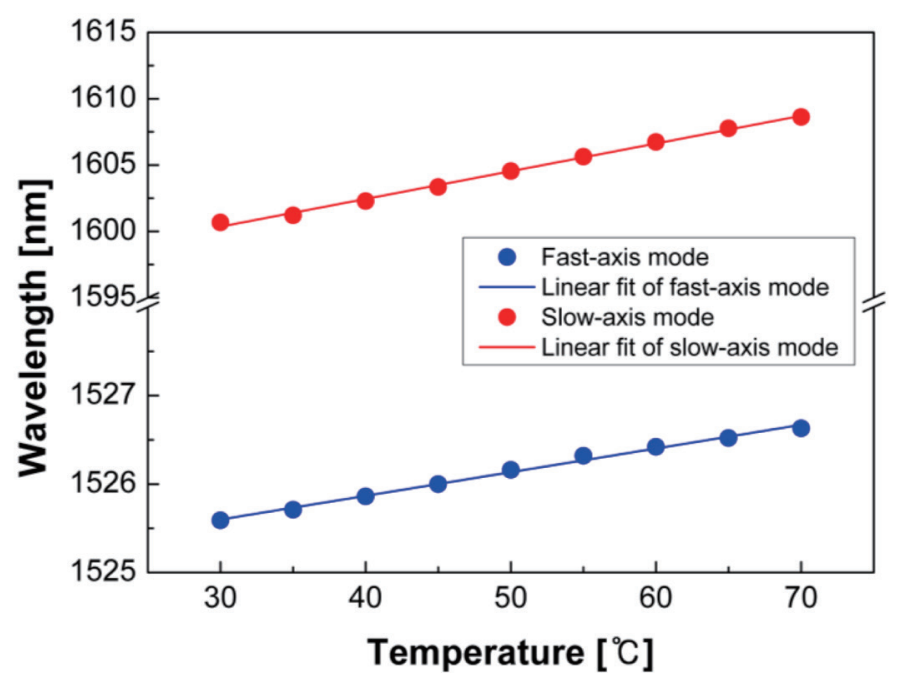

Fig. 7. (Color online) Wavelength shifts of indicator dips in fast-axis and slow-axis mode interference spectra, displayed as blue and red circles, respectively, when ambient temperature increases from 30 to $70{ }^{\circ} \mathrm{C}$ (step: $5{ }^{\circ} \mathrm{C}$ ). Solid lines are linear fits obtained with linear regression. 
$R^{2}$ values were calculated as $\sim 0.993$ and $\sim 0.996$, respectively, which implies that the fastaxis and slow-axis mode spectra have sufficiently linear temperature responses. Since the water level and temperature responses of both interference spectra of the in-fiber MZI are linear and independent, it is possible to simultaneously measure changes in both the water level and ambient temperature by incorporating a matrix that relates these water level and temperature changes with the wavelength shifts of the indicator dips. Because the water level and temperature responses of both interference spectra of the in-fiber MZI are linear, the wavelength shifts of the fast-axis and slow-axis mode interference spectra, that is, $\Delta \lambda($ LHP) and $\Delta \lambda(\mathrm{LVP})$, can be expressed as the matrix equation.

$$
\left[\begin{array}{c}
\Delta \lambda(\mathrm{LHP}) \\
\Delta \lambda(\mathrm{LVP})
\end{array}\right]=\left[\begin{array}{cc}
S_{W L, L H P}(=-37.7 \mathrm{pm} / \mathrm{mm}) & S_{T, L H P}\left(=26.8 \mathrm{pm} /{ }^{\circ} \mathrm{C}\right) \\
S_{W L, L V P}(=-137.0 \mathrm{pm} / \mathrm{mm}) & S_{T, L V P}\left(=209.0 \mathrm{pm} /{ }^{\circ} \mathrm{C}\right)
\end{array}\right]\left[\begin{array}{l}
\Delta D \\
\Delta T
\end{array}\right]
$$

Here, $\Delta D$ and $\Delta T$ are changes in water level and ambient temperature, respectively. The clear difference between $S_{W L, L H P} / S_{T, L H P}$ (approximately $-1.407{ }^{\circ} \mathrm{C} / \mathrm{mm}$ ) and $S_{W L, L V P} / S_{T, L V P}$ (approximately $-0.656{ }^{\circ} \mathrm{C} / \mathrm{mm}$ ) confirms that the response of the fast-axis mode spectrum has an independent relationship with that of the slow-axis one. Owing to this independence, Eq. (10) has a unique solution. That is, water level and temperature variations ( $\triangle D$ and $\Delta T$, respectively) applied to the in-fiber MZI can be simultaneously determined using $\Delta \lambda$ (LHP) and $\Delta \lambda$ (LVP).

\section{Conclusions}

We experimentally demonstrated a temperature-independent OFLLS based on a 83.4-mmlong in-fiber MZI (i.e., cascaded PM-LPFGs). The cascaded PM-LPFGs fabricated using a $\mathrm{CO}_{2}$ laser has a 55.0-mm-long GFFS, which serves as a sensitive area for water level measurement, between two PM-LPFGs, each of which has a grating period of $490 \mu \mathrm{m}$ and a grating length of $14.2 \mathrm{~mm}$. If the SRI of the GFFS is changed by an increase in the water level or the ambient temperature is varied, the in-fiber interference created by the cascaded PM-LPFGs spectrally shifts. This SRI- or temperature-induced wavelength shift is dependent on the input polarization of light introduced into the cascaded PM-LPFGs. For two orthogonally polarized input signals (i.e., LHP and LVP input signals), the water level sensitivity was measured as approximately -37.7 and $-137.0 \mathrm{pm} / \mathrm{mm}$ with adjusted $R^{2}$ values of $\sim 0.999$ and $\sim 0.995$ in a water level measurement range from 0 to $55 \mathrm{~mm}$, respectively. Moreover, the temperature sensitivities at LHP and LVP input signals were measured as $\sim 26.8$ and $\sim 209.0 \mathrm{pm} / \mathrm{mm}$ with adjusted $R^{2}$ values of $\sim 0.993$ and $\sim 0.996$ in a temperature measurement range from 30 to $70{ }^{\circ} \mathrm{C}$, respectively. Linear and independent responses of the fast-axis and slow-axis mode interference spectra to water level and ambient temperature changes enable our sensor to simultaneously measure water level and ambient the temperature variations. Our OFLLS can offer a costeffective way for the temperature-independent measurement of the water level with high linearity. 


\section{Acknowledgments}

This research was supported by the Basic Science Research Program through the National Research Foundation (NRF) of Korea funded by the Ministry of Education (2019R1I1A3A01046232).

\section{References}

1 K. O. Hill, Y. Fujii, D. C. Johnson, and B. S. Kawasaki: Appl. Phys. Lett. 32 (1978) 647. https://doi. org/10.1063/1.89881

2 W. W. Morey, J. R. Dunphy, and G. Meltz: Fiber Integr. Opt. 10 (1991) 351. https://doi. org $/ 10.1080 / 01468039108201715$

3 J. Jung, H. Nam, B. Lee, J. O. Byun, and N. S. Kim: Appl. Opt. 38 (1999) 38. https://doi.org/10.1364/ AO. 38.002752

4 K. O. Hill and G. Meltz: J. Lightwave Technol. 15 (1997) 1263. https://doi.org/10.1109/50.618320

5 W. Liang, Y. Huang, Y. Xu, R. K. Lee, and A. Yariv: Appl. Phys. Lett. 86 (2005) 151122. https://doi. $\operatorname{org} / 10.1063 / 1.1904716$

6 K. R. Sohn and J. H. Shim: Sens. Act. A 152 (2009) 248. https://doi.org/10.1016/j.sna.2009.04.003

7 Y. Dong, S. Xiao, H. Xiao, J. Liu, C. Sun, and S. Jian: IEEE Photon. Technol. Lett. 29 (2017) 1067. https://doi. org/10.1109/LPT.2017.2700623

8 W. Xu, J. Wang, J. Zhao, C. Zhang, J. Shi, X. Yang, and J. Yao: IEEE Sens. J. 17 (2017) 1347. https://doi. org/10.1109/JSEN.2016.2629488

9 B. Yun, N. Chen, and Y. Cui: IEEE Photon. Technol. Lett. 19 (2007) 1747. https://doi.org/10.1109/ LPT.2007.905093

10 D. Xiaowei and Z. Ruifeng: Opt. Laser Technol. 42 (2010) 214. https://doi.org/10.1016/j.optlastec.2009.06.015

11 H. Y. Chang, Y. C. Chang, H. J. Sheng, M. Y. Fu, W. F. Liu, and R. Kashyap: IEEE Photon. Technol. Lett. 28 (2016) 268. https://doi.org/10.1109/LPT.2015.2494611

12 A. M. Vengsarkar, P. J. Lemaire, J. B. Judkins, V. Bhatia, T. Erdogan, and J. E. Sipe: J. Lightwave Technol. 14 (1996) 14. https://doi.org/10.1109/50.476137

13 H. J. Patrick, A. D. Kersey, and F. Bucholtz: J. Lightwave Technol. 16 (1998) 1606. https://doi. org $/ 10.1109 / 50.712243$

14 S. Khaliq, S. W. James, and R. P. Tatam: Opt. Lett. 26 (2001) 1224. https://doi.org/10.1364/OL.26.001224

15 H. Fu, X. Shu, A. Zhang, W. Liu, L. Zhang, S. He, and I. Bennion: IEEE Sens. J. 11 (2011) 2878. https://doi. org/10.1109/JSEN.2011.2145416

16 H. Xue, Z. Xu, H. Chen, Y. Yang, J. You, J. Yan, H. Fu, and D. Zhang: Meas. Sci. Technol. 26 (2015) 37001. https://doi.org/10.1088/0957-0233/26/3/037001

17 K. J. Han, Y. W. Lee, J. Kwon, S. Roh, J. Jung, and B. Lee: IEEE Photon. Technol. Lett. 16 (2004) 2114. https:// doi.org/10.1109/LPT.2004.833081

18 T. Allsop, D. J. Webb, and I. Bennion: Opt. Fiber Technol. 9 (2003) 210. https://doi.org/10.1016/S10685200(03)00028-2

\section{About the Authors}

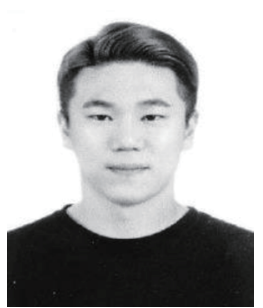

Sungwook Choi received his B.S. and M.S. degrees from the School of Electrical Engineering and the Interdisciplinary Program of Biomedical Mechanical and Electrical Engineering, Pukyong National University, Busan, South Korea, in 2015 and 2017, respectively, where he is currently pursuing his Ph.D. degree under the Interdisciplinary Program of Biomedical Mechanical and Electrical Engineering. His research interests include optical fiber sensors and switching devices based on oxide semiconductor thin films. (leewan777@gmail.com) 


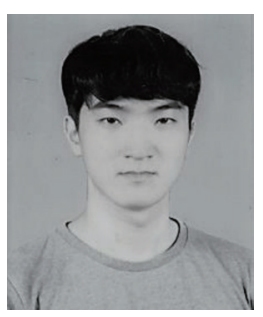

Do Kyung Kim received his B.S. degree from the School of Electrical Engineering, Pukyong National University, Busan, South Korea in 2017, where he is currently pursuing his master's degree under the Interdisciplinary Program of Biomedical Mechanical and Electrical Engineering. His research interests include optical fiber devices for optical sensors and filters.

(dosokre@naver.com)

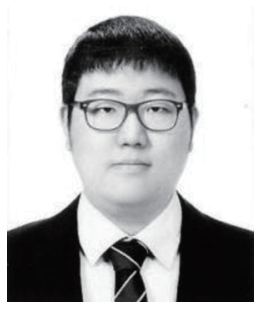

Jihoon Kim received his B.S. and M.S. degrees from the School of Electrical Engineering, Pukyong National University, Busan, South Korea, in 2013 and 2015, respectively, where he is currently pursuing his Ph.D. degree. His current research interests include current switching in semiconductor thin films based on photo-induced phase transition and the areas of optical devices for optical sensors and communications, such as optical fiber gratings and filters.(ghkim613@gmail.com)

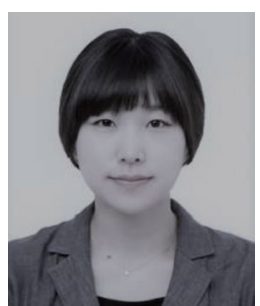

Seul-Lee Lee received her B.S. and M.S. degrees from the School of Electrical Engineering, Pukyong National University, Busan, South Korea, in 2012 and 2014, respectively, where she is currently pursuing her Ph.D. degree under the Interdisciplinary Program of Biomedical Mechanical and Electrical Engineering. Her research interests include the areas of optical fiber devices for optical sensors and communications, such as optical fiber gratings and optical fiber lasers. (kls1116@hanmail.net)

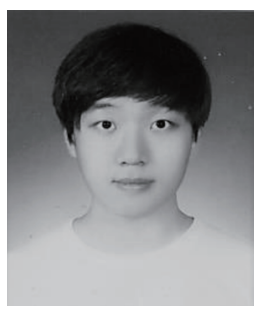

Min Seok Kim received his B.S. degree from the School of Electrical Engineering, Pukyong National University, Busan, South Korea in 2017, where he is currently pursuing his master's degree under the Interdisciplinary Program of Biomedical Mechanical and Electrical Engineering. His research interests include optical fiber lasers, optical fiber sensors, and electronic sensors based on thin film semiconductors. (doragee91@naver.com)

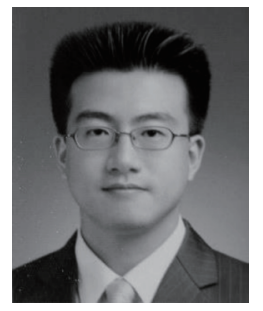

Yong Wook Lee received his B.S., M.S., and Ph.D. degrees from the School of Electrical Engineering, Seoul National University, Seoul, South Korea, in 1998, 2000, and 2004, respectively. From 2004 to 2008, he was a Senior Researcher with the Electronics and Telecommunications Research Institute, Daejeon, South Korea. He is currently a professor with the School of Electrical Engineering, Pukyong National University, Busan, South Korea. $\mathrm{He}$ is the author of more than 100 journal papers. His research interests include the areas of optical devices for optical sensors and communications, such as optical fiber gratings, optical filters, optical amplifiers, and optical switching in semiconductor thin films based on photo-induced phase transition. (yongwook@pknu.ac.kr) 